\title{
ROUTEING ON TREES
}

\author{
MARIA DEIJFEN, ${ }^{*}$ Stockholm University \\ NINA GANTERT, ${ }^{* *}$ Technische Universität München
}

\begin{abstract}
We consider three different schemes for signal routeing on a tree. The vertices of the tree represent transceivers that can transmit and receive signals, and are equipped with independent and identically distributed weights representing the strength of the transceivers. The edges of the tree are also equipped with independent and identically distributed weights, representing the costs for passing the edges. For each one of our schemes, we derive sharp conditions on the distributions of the vertex weights and the edge weights that determine when the root can transmit a signal over arbitrarily large distances.
\end{abstract}

Keywords: Trees; transmission; first passage percolation; branching random walk; Markov chain

2010 Mathematics Subject Classification: Primary 60K37

Secondary 60J80; 60J10

\section{Introduction}

Let $\mathcal{T}$ be a rooted infinite $m$-ary tree and assign independent and identically distributed (i.i.d.) weights $\left\{R_{x}\right\}$ to the vertices of $\mathcal{T}$ and i.i.d. weights $\left\{C_{e}\right\}$ to the edges. Assume that $\left\{R_{x}\right\}$ is independent of $\left\{C_{e}\right\}$. We think of the vertices as representing transceivers that can receive and transmit signals. The vertex weights represent the strength or range of the transceivers and the edge weights represent the cost or resistance when traversing the edges. We study three different schemes for signal routeing in $\mathcal{T}$ and, for each of these schemes, we investigate when the root can transmit a signal over arbitrarily large distances. More specifically, write $O$ for the set of vertices that are reached by a signal transmitted by the root, and say that a scheme can transmit indefinitely if $|O|=\infty$ with positive probability. Our main results are sharp conditions on the distributions of $R$ and $C$ that determine when the respective routeing schemes can transmit indefinitely. Here and throughout the paper, $R$ and $C$ denote random variables with the laws of $R_{x}$ and $C_{e}$, respectively.

Write $\Gamma_{x, y}$ for the path between the vertices $x$ and $y$ in $\mathcal{T}$, and write $y>x$ if $y$ is located in the subtree below $x$ in $\mathcal{T}$ (so that $y$ is hence further away from the root than $x$ ). For each vertex $x$, let $\Lambda_{x}$ be the set of all vertices $y$ in the subtree below $x$ for which the total cost of the path from $x$ to $y$ does not exceed the range of $x$, that is,

$$
\Lambda_{x}=\left\{y>x: \sum_{e \in \Gamma_{x, y}} C_{e} \leq R_{x}\right\} .
$$

Received 8 May 2015; revision received 27 June 2015.

* Postal address: Department of Mathematics, Stockholm University, 10691 Stockholm, Sweden.

Email address: mia@math.su.se

** Postal address: Technische Universität München, Boltzmannstr. 3, 85748 Garching, Germany. 
We say that the vertices in $\Lambda_{x}$ are within the range of $x$. The schemes that we will consider are now defined as follows.

Complete routeing. The root 0 first transmits the signal to all vertices in $\Lambda_{0}$. In the next step, each vertex $x \in \Lambda_{0}$ forwards the signal to all vertices in $\Lambda_{x}$, and the signal is then forwarded according to the same rule by each new vertex that is reached by it. Note that edges leading back towards the root are not used in the forwarding process, that is, the transceivers do not forward the signal through the same edge that the signal arrived from. This simplifies the analysis since it implies that whether a signal reaches a vertex $y$ or not is determined only by the configuration on the path between 0 and $y$.

Boundary routeing. For a connected subset $\Omega$ of the vertices in $\mathcal{T}$, with $0 \in \Omega$, let $\partial \Omega$ denote the set of vertices in $\Omega$ that have at least one child that is not in $\Omega$. The transmission is initiated in that the root 0 transmits the signal to all vertices in $\Lambda_{0}$, and the signal is then forwarded stepwise: if the set of vertices that have received the signal after a certain step is $\Omega$, then, in the next step, the signal is forwarded by each $x \in \partial \Omega$ to all vertices $y$ in $\Lambda_{x}$ such that the path between $x$ and $y$ (excluding $x$ ) contains only vertices in $\Omega^{c}$. The difference compared to complete routeing is hence that only vertices with neighbors that have not yet heard the signal forward the signal and then only in the direction of these uninformed neighbors.

Augmented routeing. For a vertex $x$ at level $k$ in $\mathcal{T}$, write $0=x_{0}, \ldots, x_{k}=x$ for the path from the root to $x$. In the last scheme, when a signal traverses an edge, its strength is reduced by the cost of the edge, and when it passes a transceiver, it is amplified by the strength of the transceiver. The signal hence reaches the vertex $x$ at level $k$ if and only if

$$
\sum_{i=0}^{n} R_{x_{i}}>\sum_{i=0}^{n} C_{\left(x_{i}, x_{i+1}\right)} \quad \text { for all } n=0,1, \ldots, k-1 .
$$

Write $O_{\text {comp }}, O_{\text {bond }}$, and $O_{\text {aug }}$ for the sets of vertices that are reached by a signal transmitted by the root using complete routeing, boundary routeing and augmented routeing, respectively. Clearly, complete routeing dominates boundary routeing in the sense that $O_{\text {bond }} \subset O_{\text {comp }}$. Furthermore, augmented routeing dominates complete routeing in the same sense. Indeed, with augmented routeing, the strength of a transceiver may be stored and used at any point in the forwarding process, while in complete routeing, a transceiver at $x$ is only effective within $\Lambda_{x}$. Hence,

$$
O_{\text {bond }} \subseteq O_{\text {comp }} \subseteq O_{\text {aug }} \text { almost surely (a.s.). }
$$

Note that, if $R \geq C$ a.s. then all three schemes can trivially transmit indefinitely, while on the other hand, if $R<C$ a.s. then a signal has no chance of spreading at all in any of the schemes. Hence, the interesting case is when $\{R \geq C\}$ has a nontrivial probability. It is then natural to investigate the possibility of infinite transmission in the schemes and to compare the schemes in this sense. For $m \geq 2$, we will derive the following conditions: augmented routeing can transmit indefinitely if and only if $\mathbb{E}\left[\mathrm{e}^{\lambda R}\right] \cdot \mathbb{E}\left[\mathrm{e}^{-\lambda C}\right]>1 / m$ for all $\lambda>0$; see Corollary 2.1. For complete routeing, there exists a constant $\beta$, defined from $R$ and $C$, such that infinite transmission is possible when $\beta<\log m$ but not when $\beta>\log m$; see Theorem 3.1. For boundary routeing, the condition is analogous to complete routeing, but with a different constant $\gamma$; see Theorem 4.1.

Are there cases when complete routeing (and thereby also augmented routeing) can transmit indefinitely but not boundary routeing? And are there cases when augmented routeing, but not boundary routeing and complete routeing, can transmit indefinitely? Furthermore, one might ask in general what happens when one or both of the variables $R$ and $C$ have power-law 
distributions. For what values of the exponents is it possible to transmit a signal over arbitrarily large distances? These questions can, and will, be answered by analyzing the conditions for infinite transmission.

The paper is organized so that augmented routeing is analyzed in Section 2, using tools related to branching random walks. Complete routeing and boundary routeing are then treated in Sections 3 and 4, respectively, by generalizing the arguments from Section 2. In each section, we also give examples and make the conditions more explicit for certain distribution types. Section 5 contains a summary, further comparison of the derived conditions, and some directions for further work. Throughout, we assume that $\{R \geq C\}$ has nontrivial probability.

\subsection{Related work}

Probability on trees has been a very active field of probability for the last decades; see, e.g. [13] for an introduction and [11] for a recent account. The work here is closely related to first-passage percolation on trees and tree-indexed Markov chains, see, e.g. [3] and [10]. We also rely on results and techniques for branching random walks, see [14]. Transceiver networks have previously been analyzed in the probability literature in the context of spatial Poisson processes, see [1], but the setup there is quite different from ours.

\section{Augmented routeing}

We begin by analyzing the augmented routeing scheme. To this end, first note that the transmission process can be represented by a process that we will identify below as a killed branching random walk: define $V_{0}=0$ for the root and then, for a vertex $y$ that is a child of $x$, let $V_{y}=V_{x}+Z_{x, y}$, where $Z_{x, y}=R_{x}-C_{(x, y)}$. This means that $V_{y}$ keeps track of the strength of the signal when it arrives at $y$. When $V_{y}$ takes on a negative value, the process dies at that location and the subtree below $y$ is declared dead.

If $m=1$, we have a random walk, killed when it takes a negative value. Hence, in this case, $\mathbb{P}\left(\left|O_{\text {aug }}\right|=\infty\right)>0$ if $\mathbb{E}[R]>\mathbb{E}[C]$ and $\mathbb{P}\left(\left|O_{\text {aug }}\right|=\infty\right)=0$ if $\mathbb{E}[R] \leq \mathbb{E}[C]$ and both expectations are finite. If $R$ and $C$ both have infinite expectations, both scenarios can happen. For the remainder of the section we assume that $m \geq 2$.

A one-dimensional, discrete-time branching random walk may be defined as follows. At the beginning, there is a single particle located at $V_{0}=0$. Its children, who form the first generation, are positioned according to a certain point process. Each of the particles in the first generation gives birth to new particles that are positioned (with respect to their birth places) according to the same point process; they form the second generation. The system then goes on according to the same mechanism. See, for instance, [14] for an account of results on this model.

In our case, each particle has $m$ children and the point process of displacements of the children of $x$ consists of $\left\{Z_{x, y}: y\right.$ child of $\left.x\right\}$. Let $\mathcal{V}$ denote the vertex set of the tree. The process starts with $V_{0}=0$ and, for a vertex $y$ that is a child of $x$, we have $V_{y}=V_{x}+Z_{x, y}$, where $\left\{Z_{x, y}: y \text { child of } x\right\}_{x \in \mathcal{V}}$ form a collection of i.i.d. random variables. Note that, unlike in 'classical' branching random walk, the displacements $\left\{Z_{x, y}: y\right.$ child of $\left.x\right\}$ are not i.i.d., since, for a fixed $x$, the term $R_{x}$ appearing in the definition of $Z_{x, y}$ is the same for all children of $x$. Nevertheless, $\left\{Z_{x, y}: y \text { child of } x\right\}_{x \in \mathcal{V}}$ are i.i.d. and, hence, $\left\{V_{y}\right\}$ fits in the more general definition of a branching random walk above.

Now kill the branching random walk at 0 , that is, whenever $V_{x}<0$, the process dies and the subtree below the vertex $x$ is declared dead. The survival probability in this killed random walk coincides with the probability of infinite transmission for augmented routeing, and we 
would hence like to obtain a condition that determines when the survival probability is strictly positive. To this end, let $Z, Z_{1}, Z_{2}, \ldots$ be i.i.d. with the same law as $Z_{x, y}$ and let $I(\cdot)$ be the right-side large deviation rate function for $Z$, defined by

$$
I(s):=\sup _{\lambda \geq 0}[\lambda s-\log \mathbb{E}[\exp (\lambda Z)]] \in[0, \infty] .
$$

Then Cramér's theorem implies that

$$
\lim _{n \rightarrow \infty} \frac{1}{n} \log \mathbb{P}\left[\frac{Z_{1}+\cdots+Z_{n}}{n} \geq s\right]=-I(s),
$$

see [5, Theorem 2.2.3], and hence $I(s)$ describes deviations 'to the right' of $s$ (note that $\lambda$ is only running through the nonnegative reals). In particular, we have $I(s)=0$ if $s \leq \mathbb{E}[Z]$.

Define

$$
s^{*}:=\sup \{s: I(s) \leq \log m\} \in(-\infty, \infty] .
$$

Note that, since $I(\cdot)$ is convex and nondecreasing, with $I(s)=0$ for $s \leq \mathbb{E}[Z]$, we have $s^{*}>0$ if and only if $I(0)<\log m$. With this at hand, we can determine when the killed branching random walk which describes the transmission process with augmented routeing has a strictly positive survival probability.

Proposition 2.1. Let $m \geq 2$. For the survival probability $\alpha=\mathbb{P}\left(\left|O_{\text {aug }}\right|=\infty\right)$ of the killed branching random walk, we have $\alpha>0$ if and only if $s^{*}>0$. In particular, $\alpha>0$ if and only if $I(0)<\log m$. Note that, if $\mathbb{E}[R-C] \geq 0$ then $I(0)=0$ so that $\alpha>0$.

Corollary 2.1. If $m \geq 2$ then $\mathbb{P}\left(\left|O_{\text {aug }}\right|=\infty\right)>0$ if and only if

$$
\mathbb{E}\left[\mathrm{e}^{\lambda R}\right] \cdot \mathbb{E}\left[\mathrm{e}^{-\lambda C}\right]>\frac{1}{m} \text { for all } \lambda \geq 0 .
$$

The proposition morally follows from Theorem 2.1 below, which goes back to Biggins, Hammersley, and Kingman, see [4], [8], and [9], respectively. For a proof, we also refer the reader to [14, Theorem 2.1]. However, we will not need Theorem 2.1 below, but will give a direct proof of Proposition 2.1 that we will then apply also for complete routeing and boundary routeing.

Theorem 2.1. (See Biggins [4], Hammersley [8], and Kingman [9].) For a branching random walk $\left\{V_{x}\right\}$, we have

$$
\lim _{n \rightarrow \infty} \frac{1}{n} \max _{x \in \mathcal{V},|x|=n} V_{x}=s^{*} \quad \mathbb{P} \text {-a.s. }
$$

Proof of Proposition 2.1. The proof is based on two standard arguments, which we recall since we will use them later. We also refer the reader to [13]. We first show that the survival probability is 0 if $s^{*}<0$ by showing that

$$
\limsup _{k} \frac{1}{k} \max _{x:|x|=k} V_{x} \leq s^{*} \quad \mathbb{P} \text {-a.s. }
$$

Indeed, (2.3) implies that, if the branching random walk is killed at the 'linear barrier' $s k$ with $s>s^{*}$ (i.e. all vertices $x_{k}$ at distance $k$ from the root with $V_{x_{k}}<s k$ are removed along with all their descendants), then it will die out a.s. Our process is killed at $s=0$ and, hence, $\alpha=0$ if $s^{*}<0$. 
To establish (2.3), we will consider the probabilities that there is a vertex $x$ at distance $k$ from the root with $V_{x} \geq s^{*}+\delta$, and use a union bound. There are $m^{k}$ such vertices, and if $\mathbb{P}\left(V_{x} \geq s^{*}+\delta\right)$ decays fast enough, our probabilities will be summable. Assume that $s^{*}<\infty$ and fix $\delta>0$. Then there is $\varepsilon>0$ such that $I\left(s^{*}+\delta\right)-\varepsilon>\log m$. Take large enough $k$ such that

$$
\mathbb{P}\left[\frac{Z_{1}+\cdots+Z_{k}}{k} \geq s^{*}+\delta\right] \leq \exp \left(-k\left(I\left(s^{*}+\delta\right)-\varepsilon\right)\right) .
$$

Now, by a union bound,

$$
\mathbb{P}\left[\frac{1}{k} \max _{x:|x|=k} V_{x} \geq s^{*}+\delta\right] \leq m^{k} \mathbb{P}\left[\frac{Z_{1}+\cdots+Z_{k}}{k} \geq s^{*}+\delta\right] \leq m^{k} \exp \left(-k\left(I\left(s^{*}+\delta\right)-\varepsilon\right)\right)
$$

and using the Borel-Cantelli lemma, we conclude that

$$
\underset{k}{\lim \sup } \frac{1}{k} \max _{x:|x|=k} V_{x} \leq s^{*}+\delta \quad \mathbb{P} \text {-a.s. }
$$

Since $\delta>0$ was arbitrary, (2.3) follows from this.

To show that the survival probability is strictly positive if $s^{*}>0$, we will construct a supercritical Galton-Watson process embedded in our tree. To this end, first note that

$$
\lim _{n \rightarrow \infty} \frac{1}{n} \log \mathbb{P}\left[\frac{Z_{1}+\cdots+Z_{j}}{j} \geq s, j=0,1, \ldots, n\right]=-I(s) ;
$$

see [12] or [5, Theorem 5.1.2]. Fix $s<s^{*}$. Since $I$ is a convex function which is strictly convex on $\{x: I(x) \in(0, \infty)\}$, we can pick $\delta>0$ such that $I(s)<\log m-\delta$. Consider an embedded Galton-Watson process consisting of all vertices at distances $k, 2 k, 3 k, \ldots$ from the root such that the path of the branching random walk between the vertex (at distance $i k$ from the root, say) and its predecessor (at distance $(i-1) k$ from the root) stays strictly above $\ell s$ at distance $\ell=(i-1) k+j(j=0,1, \ldots, k)$ from the root. Take large enough $k$ such that

$$
\mathbb{P}\left[\frac{Z_{1}+\cdots+Z_{j}}{j} \geq s, j=0,1, \ldots, k\right] \geq \exp (-k(I(s)+\delta)) .
$$

Then the embedded Galton-Watson process has expected offspring at least $\exp (-k(I)+$ $\delta)) m^{k}>1$, and therefore it has a strictly positive survival probability. An infinite path $0=$ $x_{0}, x_{1}, x_{2} \ldots$ from the root, where $x_{i}$ is a child of $x_{i-1}$, for all $i$, is called a ray. The above argument shows that for $s<s^{*}$, we have

$$
\mathbb{P}\left(\text { there exists a ray }\left\{x_{n}\right\} \text { with } V_{x_{n}} \geq n s \text { for all } n\right)>0 \text {. }
$$

In particular, if the branching random walk is killed at the 'linear barrier' $s k$, with $s<s^{*}$, it survives with positive probability. Hence, $\alpha>0$ if $s^{*}>0$, since our process is killed at $s=0$.

Finally, we consider the critical case $s^{*}=0$. This requires a refinement of the argument for the case when $s^{*}<0$ : assume that $s^{*}=0$, so that $I(0)=\log m$. Then, by the Bahadur-Rao theorem (see [5, Theorem 3.7.4]), there is a constant $c>0$ such that, for all $k$,

$$
\mathbb{P}\left[\frac{Z_{1}+\cdots+Z_{k}}{k} \geq 0\right] \leq \frac{c}{\sqrt{k}} \exp (-k(I(0)) .
$$


Now consider the probability that there is a vertex $x$ at distance $k$ from the root with $V_{x} \geq 0$. There are $m^{k}$ such vertices and, using a union bound, we obtain

$$
\mathbb{P}\left[\frac{1}{k} \max _{x:|x|=k} V_{x} \geq 0\right] \leq m^{k} P\left[\frac{Z_{1}+\cdots+Z_{k}}{k} \geq 0\right] \leq \frac{c}{\sqrt{k}} .
$$

We conclude, using the Borel-Cantelli lemma along the subsequence $k^{4}(k=1,2, \ldots)$, that

$$
\frac{1}{k^{4}} \max _{x:|x|=k^{4}} V_{x} \geq 0 \text { only for finitely many } k \quad \mathbb{P} \text {-a.s. }
$$

This implies that $\alpha=0$. In fact, much more is known: a 'nearly optimal' ray consists of vertices $x_{k}$ with $V_{x_{k}} \geq\left(s^{*}-\varepsilon\right) k$ for all $k$ and in [7, Theorem 1.2] it is shown that the probability that a nearly optimal ray exists goes to 0 as $\varepsilon \rightarrow 0$.

Remark 2.1. Let $\partial \mathcal{T}$ denote the boundary of the tree which is defined as the set of all rays in the tree. One can use a $0-1$ law as in [13, Proposition 3.2] to conclude from (2.4) that for $s<s^{*}$, we have

$$
\mathbb{P}\left(\sup _{\xi \in \partial \mathcal{T}} \liminf _{x_{k} \in \xi,\left|x_{k}\right|=k} \frac{1}{k} V_{x_{k}} \geq s\right)=1,
$$

which implies that

$$
\mathbb{P}\left(\sup _{\xi \in \partial \mathcal{T}} \liminf _{x_{k} \in \xi,\left|x_{k}\right|=k} \frac{1}{k} V_{x_{k}} \geq s^{*}\right)=1 .
$$

Now, Theorem 2.1 follows, in our setup, from (2.3) and (2.5).

Example 2.1. Let $R$ and $C$ be Poisson distributed with mean $\mu_{R}$ and $\mu_{C}$, respectively. Then

$$
\log \mathbb{E}\left[\mathrm{e}^{\lambda(R-C)}\right]=\left(\mathrm{e}^{\lambda}-1\right) \mu_{R}+\left(\mathrm{e}^{-\lambda}-1\right) \mu_{C}
$$

and (2.1) yields, after an easy calculation, that infinite transmission is possible if and only if $\sqrt{\mu_{C}}-\sqrt{\mu_{R}}<\sqrt{\log m}$.

Example 2.2. Let $C \equiv 1$ and assume that a transceiver is either functioning with range 1 (probability $r_{1} \neq 1$ ) or nonfunctioning with range 0 (probability $r_{0}$ ). Then

$$
\mathbb{E}\left[\mathrm{e}^{\lambda R}\right] \mathbb{E}\left[\mathrm{e}^{-\lambda C}\right]=r_{0} \mathrm{e}^{-\lambda}+r_{1}
$$

and we see that (2.1) is satisfied if and only if $r_{1}>1 / m$. Next assume that a functioning transceiver has range 2 (probability $r_{2}=1-r_{0}$ ). We apply Proposition 2.1 , calculating $I(0)=0$ if $r_{2} \geq \frac{1}{2}$ and $I(0)=-\log \left(2 \sqrt{r_{2}\left(1-r_{2}\right)}\right)$ otherwise, and obtain that either $r_{2} \geq \frac{1}{2}$ or $r_{2}\left(1-r_{2}\right)>\left(4 m^{2}\right)^{-1}$. Hence, infinite transmission is possible if and only if

$$
r_{2}>\frac{1}{2}\left(1-\sqrt{1-\frac{1}{m^{2}}}\right) .
$$

Example 2.3. Consider the case with $R \equiv 1$ and $C \in\{0,2\}$, with $\mathbb{P}(C=0)=p_{0}$ and $\mathbb{P}(C=2)=p_{2}$. This is equivalent to the previous example with $r_{2}=p_{0}$ and $r_{0}=p_{2}$ in the sense that the effect of passing a transceiver and a consecutive edge is that either the signal strength is increased by 1 (probability $p_{0}$ ) or decreased by 1 (probability $p_{2}$ ). It follows that infinite transmission is possible if and only if

$$
p_{0}>\frac{1}{2}\left(1-\sqrt{1-\frac{1}{m^{2}}}\right) \text {. }
$$


Example 2.4. Set $C \equiv 1$ and let $R$ be Poisson distributed with mean $\mu$. Then (2.1) is equivalent to

$$
\left(\mathrm{e}^{\lambda}-1\right) \mu-\lambda+\log m>0 \text { for all } \lambda \geq 0 .
$$

The minimal value is attained for $\lambda=-\log \mu$, and hence (2.6) holds if $\mu>1$ or $1-\mu+$ $\log (m \mu)>0$. For $m=2$, we obtain numerically that infinite transmission is possible if and only if $\mu>0.23$.

\section{Complete routeing}

For complete routeing, the transmission process can be described by a process $\left\{W_{y}\right\}$ that keeps track of the remaining range of a signal from the root when it reaches $y$ and is defined as follows. Set $W_{0}=0$ for the root and then, for a vertex $y$ that is a child of $x$ in the tree, let

$$
W_{y}= \begin{cases}R_{x}-C_{(x, y)} & \text { if } R_{x}>W_{x}, \\ W_{x}-C_{(x, y)} & \text { otherwise }\end{cases}
$$

Indeed, if $R_{x}>W_{x}$ then the range of the transceiver at $x$ is larger than the remaining range of the routed signal at $x$. Hence, by the definition of the scheme, the remaining range at a given child $y$ of $x$ is $R_{x}$ minus the cost $C_{(x, y)}$ of the edge $(x, y)$. If $R_{x} \leq W_{x}$ on the other hand, then the transceiver at $x$ does not increase the remaining range, and the remaining range at a given child $y$ is therefore $W_{x}$ minus the cost $C_{(x, y)}$ of the edge $(x, y)$. When $W_{y}$ takes on a negative value, the process dies at that location and all vertices in the subtree below $y$ are assigned the value $\Gamma$, where $\Gamma$ is a cemetery state.

If $m=1$, we have a Markov chain, killed when it takes a negative value. When $R$ has bounded support, say $R \leq b$ a.s., then infinite transmission is not possible: let $c>0$ and $r<c$ be any numbers such that $\mathbb{P}(C \geq c)>0$ and $\mathbb{P}(R \leq r)>0$ (such numbers exist because $\mathbb{P}(R \geq C)<1)$. Consider a sequence of length $\lceil b /(c-r)\rceil+1$ such that the strength of each transceiver is at most $r$ while the cost of the incoming link is at least $c$. Such a sequence occurs eventually with probability 1 and, since $W_{y} \leq b$, it is not hard to see that it kills the signal. Furthermore, we see directly, or from (1.1), that infinite transmission is not possible if $\mathbb{E}[R] \leq \mathbb{E}[C]<\infty$. In the general case, we do not know if survival is possible.

For the remainder of the section assume that $m \geq 2$. The process $\left\{W_{y}\right\}$ is not a branching random walk. It is also not a tree-valued Markov chain in the sense of [2], since the values of the vertices of two children of $x$ are not chosen independently given $W_{x}$. In addition, the Markov process we are considering is not irreducible. Nevertheless, the arguments of the previous section apply and we can give conditions for a positive survival probability. To this end, let $W_{0}, W_{1}, W_{2}, \ldots$ be a Markov process with the same law as $W_{0}, W_{x_{1}}, W_{x_{2}}, \ldots$, where $x_{i}$ is a child of $x_{i-1}$. Hence, the transition mechanism is the following. Take two i.i.d. sequences $\left\{C_{i}\right\}$ and $\left\{R_{i}\right\}$ which are independent. Given $W_{i-1}$, we set $W_{i}=\Gamma$ if $W_{i-1}=\Gamma$, and if $W_{i-1} \geq 0$, we set

$$
W_{i}= \begin{cases}R_{i}-C_{i} & \text { if } R_{i}>W_{i-1} \text { and } R_{i}-C_{i} \geq 0, \\ W_{i-1}-C_{i} & \text { if } R_{i} \leq W_{i-1} \text { and } W_{i-1}-C_{i} \geq 0, \\ \Gamma & \text { otherwise. }\end{cases}
$$

Denote by $\mathbb{P}_{z}$ the probability measure associated with the Markov process started from $z \in \mathbb{R}$ (the transmission process is started from $W_{0}=0$ but in the proof of Theorem 3.1 below we need to consider arbitrary starting points). This Markov process has $\Gamma$ as an absorbing state. 
Note that, due to subadditivity, the limit

$$
-\lim _{n \rightarrow \infty} \frac{1}{n} \log \inf _{z \in \mathbb{R}^{+}} \mathbb{P}_{z}\left(W_{n} \geq 0\right)=-\lim _{n \rightarrow \infty} \frac{1}{n} \log \inf _{z \in \mathbb{R}^{+}} \mathbb{P}_{z}\left(W_{n} \neq \Gamma\right)
$$

exists. The state space of the Markov chain $\left\{W_{i}\right\}$ is (a subset of) $\{\Gamma\} \cup[0, \infty)$. We can think of the state space as an ordered set, with smallest element $\Gamma$, and we claim that

$$
\inf _{z \in \mathbb{R}^{+}} \mathbb{P}_{z}\left(W_{n} \geq 0\right)=\mathbb{P}_{0}\left(W_{n} \geq 0\right) .
$$

Indeed, using the natural coupling for two Markov chains distributed according to $\mathbb{P}_{z}$ and $\mathbb{P}_{y}$, respectively, which is to take the same sequences $\left\{C_{i}\right\}$ and $\left\{R_{i}\right\}$ in the above construction, we see that for any $z$ and $y$ with $y<z$, the law of $W_{1}$ under $\mathbb{P}_{y}$ is dominated by the law of $W_{1}$ under $\mathbb{P}_{z}$, and by induction, the law of $W_{n}$ under $\mathbb{P}_{0}$ is dominated by the law of $W_{1}$ under $\mathbb{P}_{z}$, for any $z>0$ and any $n$. We conclude that

$$
\beta:=-\lim _{n \rightarrow \infty} \frac{1}{n} \log \mathbb{P}_{0}\left(W_{n} \geq 0\right)
$$

exists and that

$$
\beta=-\lim _{n \rightarrow \infty} \frac{1}{n} \log \inf _{z \in \mathbb{R}^{+}} \mathbb{P}_{z}\left(W_{n} \geq 0\right) .
$$

In the following theorem we assert that complete routeing can transmit indefinitely if $\beta<$ $\log m$ but not if $\beta>\log m$.

Theorem 3.1. Assume that $m \geq 2$ and let $\beta$ be defined as in (3.1).

(i) If, for some subsequence $n_{k}$ of the integers with $n_{k} \rightarrow \infty$ as $k \rightarrow \infty$,

$$
\sum_{k=1}^{\infty} m^{n_{k}} \mathbb{P}_{0}\left(W_{n_{k}} \geq 0\right)<\infty
$$

then $\mathbb{P}\left(\left|O_{\text {comp }}\right|=\infty\right)=0$. In particular, (3.3) is satisfied if $\beta>\log m$.

(ii) If $\beta<\log m$ then $\mathbb{P}\left(\left|O_{\text {comp }}\right|=\infty\right)>0$.

Proof. The proof of Theorem 3.1(i) is the same as the proof of (2.3), and the proof of Theorem 3.1(ii) is the same as the proof of (2.4). Indeed, using a union bound,

$$
\mathbb{P}\left[\frac{1}{k} \max _{x:|x|=k} W_{x} \geq 0\right] \leq m^{k} \mathbb{P}\left[W_{k} \geq 0\right]
$$

and, hence, it follows from the Borel-Cantelli lemma that, if (3.3) holds then

$$
\underset{k}{\limsup } \frac{1}{k} \max _{x:|x|=k} V_{x}<0 \quad \mathbb{P} \text {-a.s. }
$$

Theorem 3.1(i) follows from this by the same argument as in the proof of (2.3).

To show Theorem 3.1(ii), we again construct an embedded Galton-Watson tree which survives with positive probability. Pick $\delta>0$ such that $\beta<\log m-\delta$ and choose large enough $k$ such that inf ${ }_{z \in \mathbb{R}^{+}} \mathbb{P}_{z}\left(W_{k} \neq \Gamma\right) \geq \exp (-k(\beta+\delta)$ ) (which is possible due to (3.2)). Consider an embedded Galton-Watson process consisting of all vertices at distances $k, 2 k, 3 k, \ldots$ from the 
root such that the path of the branching random walk between the vertex (at distance $i k$ from the root, say) and its predecessor (at distance $(i-1) k$ from the root) does not hit $\Gamma$ (note that it suffices that $W$ takes nonnegative values at the vertex since $\Gamma$ is an absorbing state). Then, the embedded Galton-Watson process has expected offspring at least $\exp (-k(\beta+\delta)) m^{k}>1$, and therefore it has a strictly positive survival probability.

Obtaining explicit expressions for the probability $\mathbb{P}\left(W_{n} \geq 0\right)$, and thereby for $\beta$, for some large class of distributions seems difficult. However, it is possible to deduce from Theorem 3.1 that infinite transmission is always possible when $R$ is a power-law or when $\mathbb{P}(C=0)>1 / \mathrm{m}$. The (simple) proofs of this are valid also for boundary routeing, and therefore we give the proofs in the next section; see Corollaries 4.1 and 4.2. Here, instead, we analyze the condition in Theorem 3.1 for some specific examples.

Example 3.1. Let $C \equiv 1$ and $R \in\{0,1\}$ with $\mathbb{P}(R=1)=r_{1}$. Then $W_{n} \geq 0$ if and only if no transceiver up to vertex $n$ is nonfunctioning with range 0 . Hence, $\mathbb{P}\left(W_{n} \geq 0\right)=r_{1}^{n}$, so that $\beta=-\log r_{1}$, which is smaller than $\log m$ when $r_{1}>1 / m$. This is the same condition as in Example 2.1, and indeed all schemes are equivalent in this case.

Example 3.2. Let $C \equiv 1$ and $R \in\{0,2\}$ with $\mathbb{P}(R=0)=r_{0}$ and $\mathbb{P}(R=2)=r_{2}=: r$. In this case, $W_{0}, W_{1}, W_{2}, \ldots$ is a Markov chain with state space $\{\Gamma, 0,1\}$ and with transition probabilities given by $p(\Gamma, \Gamma)=1, p(0, \Gamma)=r_{0}, p(0,1)=r, p(1, \Gamma)=0, p(1,0)=r_{0}$, and $p(1,1)=r$. The transition matrix can be diagonalized and has the eigenvalues $1, \frac{1}{2}(r+a)$ and $\frac{1}{2}(r-a)$, where $a=\sqrt{4 r-3 r^{2}}$. We conclude that $\beta=-\log \left(\frac{1}{2}(r+a)\right)$. Hence,

$$
\mathbb{P}\left(\left|O_{\text {comp }}\right|=\infty\right)>0 \text { if } r+\sqrt{4 r-3 r^{2}}>\frac{2}{m} .
$$

and

$$
\mathbb{P}\left(\left|O_{\text {comp }}\right|=\infty\right)=0 \quad \text { if } r+\sqrt{4 r-3 r^{2}}<\frac{2}{m} .
$$

This can be written as

$$
\mathbb{P}\left(\left|O_{\text {comp }}\right|=\infty\right)>0 \quad \text { if } r>\frac{1}{2}\left(1+\frac{1}{m}-\sqrt{1+\frac{2}{m}-\frac{3}{m^{2}}}\right)
$$

and

$$
\mathbb{P}\left(\left|O_{\text {comp }}\right|=\infty\right)=0 \quad \text { if } r<\frac{1}{2}\left(1+\frac{1}{m}-\sqrt{1+\frac{2}{m}-\frac{3}{m^{2}}}\right) .
$$

In particular, recalling the condition for augmented routeing from Example 2.3, we see that $r$ can be chosen such that infinite transmission is possible for augmented routeing, but not for complete routeing. For $m=2$ for instance, the critical value for $r$ is approximately 0.19 with complete routeing and approximately 0.067 with augmented routeing. We remark that, diagonalizing the transition matrix, one can compute that

$$
\mathbb{P}\left(W_{n} \geq 0\right)=\frac{r+a}{2 a}\left(\frac{r+a}{2}\right)^{n}+\frac{r-a}{2 a}\left(\frac{r-a}{2}\right)^{n},
$$

but this does not help to settle the critical case $\beta=\log m$, since (3.3) is not satisfied. In general, we believe that, in the critical case, both scenarios are possible depending on the distributions. 
Example 3.3. Next, we give another example where augmented routeing is strictly more powerful than complete routeing. To this end, recall Example 2.2 where is was shown that when $R \equiv 1$ and $C \in\{0,2\}$, with $\mathbb{P}(C=0)=p_{0}$, then infinite transmission is possible with augmented routeing if and only if $p_{0}>\frac{1}{2}\left(1-\sqrt{1-1 / m^{2}}\right)$. For complete routeing, we note that $W_{n} \geq 0$ if and only if no edge between the root and vertex $n$ has weight 2 . Thus, $\beta=\log p_{0}$, implying that infinite transmission is possible if $p_{0}>1 / \mathrm{m}$, but not if $p_{0}<1 / \mathrm{m}$. For $m \geq 2$ and $p_{0}=1 / 2 m$, augmented routeing can hence transmit indefinitely, but complete routeing cannot.

\section{Boundary routeing}

First note that, when $\{R \geq C\}$ has a nontrivial probability, infinite transmission is never possible with boundary routeing for $m=1$. Indeed, the tree $\mathcal{T}$ then reduces to a singly infinite path and the time until we encounter a transceiver at the boundary of the set of the informed vertices whose strength is strictly smaller than the cost of the edge to its uninformed neighbor is clearly a.s. finite. We hence restrict to $m \geq 2$.

We begin by giving an explicit condition for infinite transmission in the case when $C \equiv c$. By scaling we can take $c=1$ and it is then enough to consider integer-valued range variables $R$. Indeed, if $R$ is not integer-valued we instead work with $R^{\prime}=\lfloor R\rfloor$ and note that this gives rise to the same transmission process.

Proposition 4.1. If $C \equiv 1$ and $R$ is integer-valued with $\mathbb{P}(R=i)=r_{i}(i=0,1,2, \ldots)$, then $\mathbb{P}\left(\left|O_{\text {bond }}\right|=\infty\right)>0$ if and only if

$$
\mathbb{E}\left[m^{R}\right]>1+r_{0}
$$

Proof. The condition follows by relating the transmission process to a branching process. The ancestor of the process is the root 0 , and the offspring of a vertex $x$ is then $\partial \Lambda_{x}$, that is, the vertices that are within the range of $x$, but that have at least one child that is not within the range of $x$. The possible offspring of $x$ are the vertices at level $R_{x}$ below $x$, and since there are $m^{k}$ vertices at level $k$ below $x$, the offspring mean is $\sum_{k=1}^{\infty} m^{k} r_{k}=\mathbb{E}\left[m^{R}\right]-r_{0}$.

Example 4.1. Let $C \equiv 1$ and assume that a transceiver is either functioning with range $n$ (probability $r_{n} \neq 1$ ) or nonfunctioning with range 0 (probability $r_{0}=1-r_{n}$ ). The root can then transmit indefinitely if and only if $r_{n}>1 / m^{n}$. For $n=2$, the condition becomes $r_{2}>1 / m^{2}$, which is strictly stronger than the condition for complete routeing derived in Example 3.2. The critical value for $r_{2}$ when $m=2$, for instance, is 0.25 with boundary routeing and approximately 0.19 with complete routeing. If $r_{i}=L(i) a^{-i}$ for some slowly varying function $L(i)$ and $a<1$, then infinite transmission is possible for $a>1 / m$, while for $a<1 / m$ it depends on the precise form of the distribution.

Example 4.2. Take $C \equiv 1$ and let $R$ be Poisson distributed with mean $\gamma$. Then (4.1) translates into $\mathrm{e}^{\gamma(m-1)}>1+\mathrm{e}^{-\gamma}$, which holds for large enough $\gamma$. For $m=2$, the threshold is $\gamma=\ln (1+\sqrt{2})=0.88$. This can be compared to the condition for augmented routeing, which is $\gamma>0.23$; see Example 2.4. For larger $m$, analytical expressions for the threshold are more involved, but numerical values are easily obtained.

When the edge costs are random, a branching process approach does not work, since information on that the signal has reached a vertex $x$, but not a given child $y$, affects the distribution of $C_{(x, y)}$ in a way that is difficult to control. Also the number of uninformed 
children of $x$ carries information about $C_{(x, y)}$. However, the arguments from the previous section can be applied again to derive a general condition. To this end, we note that the transmission process can be described by a process $\left\{U_{y}\right\}$ that keeps track of the strength of a signal from the root when it reaches $y$ and is defined as follows. Set $U_{0}=0$ for the root and then, for a vertex $y$ that is a child of $x$ in the tree, let

$$
U_{y}= \begin{cases}U_{x}-C_{(x, y)} & \text { if } U_{x}-C_{(x, y)} \geq 0, \\ R_{x}-C_{(x, y)} & \text { otherwise. }\end{cases}
$$

Indeed, when $U_{x}-C_{(x, y)}$ becomes strictly negative, we have passed a vertex that is on the boundary of the informed set. The transceiver at $x$ then forwards the signal and the new balance is $R_{x}-C_{(x, y)}$. When $U_{y}$ takes on a negative value, the process dies at that location and all vertices in the subtree below $y$ are assigned the value $\Gamma$, where $\Gamma$ is a cemetery state.

Let $U_{0}, U_{1}, \ldots$ be a Markov process distributed as the above process along a given ray in the tree, that is, $\Gamma$ is an absorbing state and, if $U_{i-1} \geq 0$, the transition mechanism is

$$
U_{i}= \begin{cases}U_{i-1}-C_{i} & \text { if } U_{i-1}-C_{i} \geq 0 \\ R_{i-1}-C_{i} & \text { if } U_{i-1}-C_{i}<0 \text { and } R_{i-1}-C_{i} \geq 0 \\ \Gamma & \text { otherwise. }\end{cases}
$$

Here $\left\{R_{i}\right\}$ and $\left\{C_{i}\right\}$ are i.i.d. sequences. Let $\mathbb{P}_{z}$ denote the probability measure of the process $\left\{U_{i}\right\}$ started from $U_{0}=z$. In analogy with complete routeing, the limit

$$
-\lim _{n \rightarrow \infty} \frac{1}{n} \log \inf _{z \in \mathbb{R}^{+}} \mathbb{P}_{z}\left(U_{n} \geq 0\right)
$$

exists due to subadditivity. Furthermore, also in this case, we have

$$
\lim _{n \rightarrow \infty} \frac{1}{n} \log \inf _{z \in \mathbb{R}^{+}} \mathbb{P}_{z}\left(U_{n} \geq 0\right)=\lim _{n \rightarrow \infty} \frac{1}{n} \log \mathbb{P}_{0}\left(U_{n} \geq 0\right) .
$$

Indeed, if the chain is started from $U_{0}=z>0$, for sure it survives to the level $M_{z}=$ $\max \left\{k: \sum_{i=1}^{k} C_{i} \leq z\right\}$, and from that point the mechanism is stochastically the same as for a process started from $U_{0}=0$. Hence,

$$
\gamma:=-\lim _{n \rightarrow \infty} \frac{1}{n} \log \mathbb{P}_{0}\left(U_{n} \geq 0\right)
$$

exists and coincides with the limit in (4.2). This means that the proof of Theorem 3.1 goes through verbatim and gives an analogous criteria for infinite transmission with boundary routeing.

Theorem 4.1. Assume that $m \geq 2$ and let $\gamma$ be defined as in (4.3).

(i) If $\gamma>\log m$ then $\mathbb{P}\left(\left|O_{\text {bond }}\right|=\infty\right)=0$.

(ii) If $\gamma<\log m$ then $\mathbb{P}\left(\left|O_{\text {bond }}\right|=\infty\right)>0$.

Just as for complete routeing, it is typically difficult to find explicit expressions for $\gamma$. However, in some cases we can give sufficient conditions for $\gamma<\log m$, and hence for the possibility of infinite transmission. First, recall that a tail distribution function $\bar{F}(x)=\mathbb{P}(X>$ $x$ ) is said to be regularly varying with tail exponent $\tau-1$ if $\bar{F}(x)=x^{-(\tau-1)} L(x)$, where $x \mapsto L(x)$ is slowly varying at $\infty$ (that is, $L(a x) / L(x) \rightarrow 1$ as $x \rightarrow \infty$ for any $a>0$ ). When this is the case, we say that the random variable $X$ has a power-law distribution. 
Corollary 4.1. If $m \geq 2$ and $R$ has a power-law distribution then $\mathbb{P}\left(\left|O_{\text {bond }}\right|=\infty\right)>0$ regardless of the distribution of $C$.

Proof. Let $S_{n}=\sum_{i=1}^{n} C_{i}$. Trivially, $\mathbb{P}\left(U_{n} \geq 0\right) \geq \mathbb{P}\left(S_{n} \leq R\right)$ since the process is clearly alive at level $n$ if the total cost of a given path of length $n$ does not exceed the range of the root transceiver. For any $c>0$, we have $\mathbb{P}\left(S_{n} \leq R\right) \geq \mathbb{P}(R \geq n c) \cdot \mathbb{P}\left(S_{n} \leq n c\right)$ and trivially, $\mathbb{P}\left(S_{n} \leq n c\right) \geq \mathbb{P}(C \leq c)^{n}$. Now take $c$ such that $\mathbb{P}(C \leq c) \geq a / m$ for some $a \in(1, m)$. Then

$$
\mathbb{P}\left(S_{n} \leq R\right) \geq \mathbb{P}(R \geq n c)\left(\frac{a}{m}\right)^{n}
$$

and it follows that $\gamma<\log m$.

The tail behavior of the cost variable $C$ does not have the same role in determining the possibility of infinite transmission. For instance, it is not the case that infinite transmission is necessarily impossible if $C$ has a power-law distribution while $R$ has a distribution with an exponentially decaying tail. Instead, a sufficiently large atom at 0 for $C$ guarantees that infinite transmission is possible, regardless of the tail behavior of the distributions.

Corollary 4.2. If $\mathbb{P}(C=0) \geq 1 /$ then $\mathbb{P}\left(\left|O_{\text {bond }}\right|=\infty\right)>0$.

Proof. For any fixed $r \geq 0$, we have

$$
\mathbb{P}\left(S_{n} \leq r\right) \geq \mathbb{P}\left(\bigcap_{i=1}^{n}\left\{C_{i} \leq \frac{r}{n}\right\}\right) \geq \mathbb{P}(C=0)^{n} .
$$

Since $\mathbb{P}\left(U_{n} \geq 0\right) \geq \mathbb{P}\left(S_{n} \leq R\right)$, this implies that $\gamma<\log m$.

\section{Summary and conclusions}

We have derived conditions for infinite transmission in all three schemes. For $m \geq 2$, the conditions are as follows.

- Augmented routeing can transmit indefinitely if and only if $\mathbb{E}\left[\mathrm{e}^{\lambda R}\right] \mathbb{E}\left[\mathrm{e}^{-\lambda C}\right]>1 / m$ for all $\lambda \geq 0$.

- Let $\left\{W_{i}\right\}$ represent the transmission process with complete routeing along a given ray (see Section 4 for a precise definition) and define $\beta=-\lim _{n \rightarrow \infty}(1 / n) \log P\left(W_{n} \geq 0\right)$. Complete routeing can then transmit indefinitely if $\beta<\log m$, but not if $\beta>\log m$. At the critical point $\beta=\log m$, we believe that both scenarios are possible.

- For $m \geq 2$, boundary routeing can transmit indefinitely if $\gamma<\log m$ but not if $\gamma>\log m$, where $\gamma=-\lim _{n \rightarrow \infty}(1 / n) \log \mathbb{P}\left(U_{n} \geq 0\right)$ and $\left\{U_{i}\right\}$ represents the transmission along a given ray. When $C \equiv 1$ and $R$ is integer-valued with $\mathbb{P}(R=i)=r_{i}$, the condition becomes $\mathbb{E}\left[m^{R}\right]>1+r_{0}$.

For $m=1$, augmented routeing can transmit indefinitely if $\mathbb{E}[R]>\mathbb{E}[C]$ but not if $\mathbb{E}[R] \leq$ $\mathbb{E}[C]$ and both expectations are finite. If $R$ and $C$ both have infinite expectations, both scenarios can happen. Complete routeing cannot transmit indefinitely when $R$ has bounded support, but the general case is open. Boundary routeing cannot transmit indefinitely for any distribution.

When $R$ has a power-law distribution and $m \geq 2$, infinite transmission is always possible with all three schemes; see Corollary 4.1. The tail behavior of $C$ does not play the same role, 
since, according to Corollary 4.2, a large enough atom at 0 guarantees that infinite transmission is possible with boundary routeing (and thereby also with the other schemes), regardless of the tail behaviors.

We have given several examples of distributions where the schemes are strictly different in the sense that there are regimes for the parameters of the distributions of $R$ and $C$ where one (or two) of the schemes can transmit indefinitely, but not the other two (one); see, for example, Examples 3.2, 3.3, 4.1, and 4.2. Complete routeing and boundary routeing are trivially equivalent in some cases, for example, when $R$ is constant (see also Example 3.1). An interesting question is if the three schemes are always strictly different when this is not the case and when $R$ does not have a power-law distribution, that is, is it then always strictly easier to transmit to $\infty$ with complete routeing than with boundary routeing, and strictly easier with augmented routeing than with complete routeing? Or are there cases when the conditions coincide for (at least) two of the schemes? Answering this is complicated by the fact that the conditions for complete routeing and boundary routeing are somewhat difficult to analyze, since the probabilities $\mathbb{P}\left(W_{n} \geq 0\right)$ and $\mathbb{P}\left(U_{n} \geq 0\right)$ are typically not easy to calculate. However, when $C \equiv 1$ and $R$ is integer-valued, the conditions for boundary routeing and for augmented routeing are explicit and an easier question is if there are families of distributions of $R$ for which these conditions coincide. Below we show that the answer is no.

The condition (4.1) for infinite transmission with boundary routeing means that $r_{0}$ has to be sufficiently small. We now show that, for any distribution that satisfies (4.1), it is possible to strictly increase $r_{0}$ and still be able to transmit indefinitely with augmented routeing. To this end, let the distribution of $R$ be described by $\left\{r_{i}\right\}_{i=0}^{\infty}$, let $k=\min \left\{i \geq 1: r_{i}>0\right\}$, and take $\varepsilon \in\left(0, r_{k}\right)$. Then define $R_{\varepsilon}$ by shifting mass $\varepsilon$ from $k$ to 0 , that is, $R_{\varepsilon}$ has distribution

$$
\mathbb{P}\left(R_{\varepsilon}=i\right)= \begin{cases}r_{k}-\varepsilon & \text { if } i=k, \\ r_{0}+\varepsilon & \text { if } i=0, \\ r_{i} & \text { otherwise }\end{cases}
$$

Proposition 5.1. Let $m \geq 2$, take $C \equiv 1$, and let $R$ be integer-valued such that (4.1) holds. If $\varepsilon$ is sufficiently small then $R_{\varepsilon}$ satisfies (2.1).

Proof. For any $\lambda>0$, we have

$$
m \mathbb{E}\left[\mathrm{e}^{\lambda R_{\varepsilon}}\right] \mathbb{E}\left[\mathrm{e}^{-\lambda C}\right] \geq r_{0} m \mathrm{e}^{-\lambda}-m \mathrm{e}^{(k-1) \lambda} \varepsilon+m \sum_{i=1}^{\infty} r_{i} \mathrm{e}^{(i-1) \lambda} .
$$

First assume that $\mathrm{e}^{\lambda} \geq m$, and write $\mathrm{e}^{\lambda}=m+c_{\lambda}$, where $c_{\lambda}>0$ and $c_{\lambda} \sim \mathrm{e}^{\lambda}$ as $\lambda \rightarrow \infty$. Then for the last term in (5.1), we obtain

$$
m \sum_{i=1}^{\infty} r_{i} \mathrm{e}^{(i-1) \lambda} \geq \sum_{i=1}^{\infty} r_{i} m^{i}+m r_{k} c_{\lambda}^{k-1}>1+m r_{k} c_{\lambda}^{k-1},
$$

where the last inequality follows from (4.1). It follows that, if $\varepsilon$ is sufficiently small then $m \mathbb{E}\left[\mathrm{e}^{\lambda R_{\varepsilon}}\right] \mathbb{E}\left[\mathrm{e}^{-\lambda C}\right]>1$ for all $\lambda$ such that $\mathrm{e}^{\lambda} \geq m$. Next assume that $\mathrm{e}^{\lambda}<m$ so that $\mathrm{e}^{-\lambda}>1 / m$. Trivially,

$$
m \sum_{i=1}^{\infty} r_{i} \mathrm{e}^{(i-1) \lambda} \geq m\left(1-r_{0}\right)
$$


and, hence, the right-hand side of (5.1) is bounded from below by

$$
r_{0}-m \mathrm{e}^{(k-1) \lambda} \varepsilon+m\left(1-r_{0}\right),
$$

which is larger than 1 for all $\lambda$ in the specified range if $\varepsilon$ is sufficiently small.

A possible continuation of the current work would be to investigate time dynamics of the transmission schemes under various rules for the transmission times. Conditionally on the event that the signal does not die, what is the asymptotic speed of the transmission? Are there setups when a scheme has a very small (large) probability of transmitting to infinity, but where the speed of transmission conditionally on survival is large (small)? Yet another question to investigate is when the root can hear a signal from infinitely far away. Do the conditions on $R$ and $C$ for this coincide with the conditions for infinite transmission? For all three routeing schemes, the probability that the root can transmit to a given vertex $x$ at level $n$ is of course the same as the probability that $x$ can transmit to the root. However, the dependence structure for the events \{root can transmit to vertex $i$ at level $n\}_{i=1}^{m^{n}}$ and \{vertex $i$ at level $n$ can transmit to the root $\}_{i=1}^{m^{n}}$ is different, and hence the conditions could possibly be different. In [6], this issue was analyzed for a related problem in the context of a spatial Poisson process.

\section{Acknowledgements}

We thank Silke Rolles and Anita Winter for inviting us to the 'Women in Probability' workshop, July 2010 at the Technische Universität München, where this work was initiated. The work of MD was supported in part by the Bank of Sweden Tercentenary Foundation and the Swedish Research Council.

\section{References}

[1] Balister, P., Bollobás, B. And Walters, M. (2009). Random transceiver networks. Adv. Appl. Prob. 41, 323-343.

[2] Benjamini, I. and Peres, Y. (1994). Markov chains indexed by trees. Ann. Prob. 22, 219-243.

[3] Benjamini, I. And Peres, Y. (1994). Tree-indexed random walks on groups and first passage percolation. Prob. Theory Relat. Fields 98, 91-112.

[4] Biggins, J. D. (1976). The first- and last-birth problems for a multitype age-dependent branching process. Adv. Appl. Prob. 8, 446-459.

[5] Dembo, A. And Zeitouni, O. (1998). Large Deviations Techniques and Applications (Appl. Math. New York 38), 2nd edn. Springer, New York.

[6] Dousse, O. (2012). Percolation in directed random geometric graphs. In Proc. IEEE Internat. Symp. Inf. Theory, IEEE, New York, pp. 601-605.

[7] Gantert, N., Hu, Y. AND Shi, Z. (2011). Asymptotics for the survival probability in a killed branching random walk. Ann. Inst. H. Poincaré Prob. Statist. 47, 111-129.

[8] Hammersley, J. M. (1974). Postulates for subadditive processes. Ann. Prob. 2, 652-680.

[9] Kingman, J. F. C. (1975). The first birth problem for an age-dependent branching process. Ann. Prob. 3, $790-801$.

[10] Lyons, R. And Pemantle, R. (1992). Random walk in a random environment and first-passage percolation on trees. Ann. Prob. 20, 125-136. (Correction: 31 (2003), 528-529.)

[11] Lyons, R. And Peres, Y. Probability on trees and networks. Available at http://mypage.iu.edu/ rdlyons/ prbtree/prbtree.html.

[12] Mogul'skĭ̌, A. A. (1977). Large deviations for trajectories of multi-dimensional random walks. Theory Prob. Appl. 21, 300-315.

[13] Peres, Y. (1999). Probability on trees: an introductory climb. In Lectures on Probability Theory and Statistics (Lecture Notes Math. 1717), Springer, Berlin, pp. 193-280

[14] SHI, Z. (2011). Random walks and trees. ESAIM Proc. 31, 1-39. 